\title{
Importance and estimation of mismatch uncertainty for RF parameters in calibration laboratories
}

\author{
K. Patel ${ }^{1, \star}$ and P.S. Negi ${ }^{2}$ \\ 1 Amorphous and Microcrystalline Silicon Solar Cells, CSIR-National Physical Laboratory, New Delhi, India \\ 2 Electrical Standards, CSIR-National Physical Laboratory, New Delhi, India
}

Received: 17 March 2012 / Accepted: 24 March 2012

\begin{abstract}
A number of calibration techniques are used in Radio Frequency (RF) calibration for power, attenuation and impedance parameters. Mismatch uncertainty plays a major role in these measurements and in estimation of combined uncertainty according to ISO/IEC 17025:2005 and GUM document. Formulas to evaluate the mismatch uncertainty in terms of reflection coefficient, voltage standing wave ratio and scattering parameters have been evaluated earlier and are being used in RF calibration. The present paper summarizes the mismatch formulas previously studied and the modified ones suitable to the measurement system and techniques available today. For power and attenuation calibration in the particular frequency range, an establishment of traceability for one-port measurement in the same frequency range is required. The methods to reduce and control this uncertainty component and their limitations are also discussed for best measurements practices in the calibration laboratories.
\end{abstract}

Keywords: Mismatch uncertainty; RF attenuation; RF power; impedance traceability; vector network analyzer

\section{Introduction}

For maximum transfer of the power from any RF source, with minimum heating from the reflected power, the total input impedance of the component connected to the source must be equal to the internal impedance of the source. So a perfect impedance match is necessary between the source, components and receiver for a lossless transmission of the signal power. The power loss due to mismatch is the mismatch loss, which is distributed in terms of return loss and heat loss [1]. Mismatch loss in transmission line theory is the amount of power expressed in decibels that will not be available on the output due to impedance mismatches and reflections. In other words, the mismatch loss represents the amount of power wasted in the system. One of the most important and dominating factors in the microwave measurements is the impedance mismatch, which should be minimum to satisfy the desirable maximum power transfer theorem. Impedance matching is an important part of RF system design; however, there is always be some degree of mismatch loss in practice. A transmission line that is properly terminated with the same impedance as that of the characteristic impedance of the transmission line, will have no reflections and therefore no mismatch loss. In real systems, relatively a little loss is due to mismatch loss and is often of the order of $1 \mathrm{~dB}[2]$.

^ Correspondence: kpatel@nplindia.org
The impedance mismatch is expressed in terms of reflection coefficient $\Gamma$, voltage standing wave ratio $(V S W R$ ) and return loss $(R L)$ and these quantities are interrelated. So a perfect match can be represented by the range $0.005 \leqslant \Gamma \geqslant 0.0,1.01 \leqslant V S W R \geqslant 1.0$ and $R L \geqslant 40 \mathrm{~dB}$, which is difficult to achieve in reality. The main cause of the mismatch is the deviation in the connecting port dimensions from the standard dimensions for device-under-test (DUT) either in coaxial or waveguide configuration [3-5]. For example, in coaxial systems an impedance of $50 \Omega$ is taken as a standard value and manufacturer tries to get this value on their products. Getting $50 \Omega$ is almost impossible on products and also on the signal source and measuring equipment, thus there is always an existence of mismatch. The effective contribution of mismatch depends on the manufacturing limitations and human handling capabilities for DUTs and microwave systems. If there are two or more components in cascade, the resultant mismatch loss is not only due to the mismatches from the individual components, but also from the reflections from each component and their combination. The overall mismatch loss cannot be calculated by just adding up the individual loss contributions from each component. The difference between the sum of the mismatch loss in each component and total mismatch loss due to the interactions of the reflections is known as mismatch error [6-8]. Depending on how the multiple reflections combine, the overall system loss may be lower 
or higher than the sum of the mismatch loss from each component. The uncertainty associated in the evaluation of the mismatch error is known as the mismatch uncertainty and expressed in $\mathrm{dB}$. The mismatch uncertainty is expressed and calculated in terms of impedance which may again re-written in terms of $\Gamma, V S W R, R L$ and scattering parameters $\left(S_{n n}, S_{m n}, m=1,2 \ldots, n=1,2 \ldots\right)[9-11]$.

\subsection{Importance of mismatch uncertainty for calibration laboratories}

In the calibration laboratories, power, attenuation and impedance are important parameters against frequency and measured to fully characterize any passive and active components. These laboratories have established and implemented the quality system and calibration facilities in accordance with the ISO/IEC 17025:2005 [12-15]. The direct, differential or null and built-up measurement techniques have already been established in such laboratories to provide the RF calibrations in the designated frequency range. The detail operation, advantages and limitations of using these techniques are well studied and upgraded in $[6,7,16]$ and $[17,18]$. The working or transfer standards for RF power parameter are generally thermistor mount, bolometers, RF transfer standards while the measuring equipment are power meter along with sensor and spectrum analyzer, etc. For attenuation parameter, fixed and variable attenuators in coaxial and waveguide configurations are served as DUTs and measuring receivers are used as transfer equipment. Thus the mismatch developed at one port for power measurements whereas it has been at two ports in attenuation measurements. The effect of the mismatch can be judged by estimating the mismatch uncertainty in RF measurements for one-port and twoport components differently. Every calibration is associated with an uncertainty value and uncertainties due to mismatch become an important contributor in the overall uncertainty analysis especially above $10 \mathrm{MHz}$.

The mismatch uncertainty is the systemic component of uncertainty in the power and attenuation measurements. The corrections for the mismatch could not be applied because the actual phases of the reflection coefficients are not known and vary with frequency and connector types. Thus the amount of residual mismatch not only limits the range of the microwave power and attenuation, but also the precise measurements at microwave frequencies, i.e., calibration. In general, the signal feed system and detector system develop capacitors and inductors as distributed components with reactance and a little resistance at RF and microwave frequencies, i.e., they have an impedance too. Thus, a need to make accurate impedance measurements has come into picture to study the effects of these components with greater knowledge and enlighten in the actual measurements. This indicates that the RF impedance lies in the complex plane, which differs from an ordinary plane only in the fact that multiplying and dividing complex numbers will give another complex number in a plane. Thus a complex measurement of the reflection

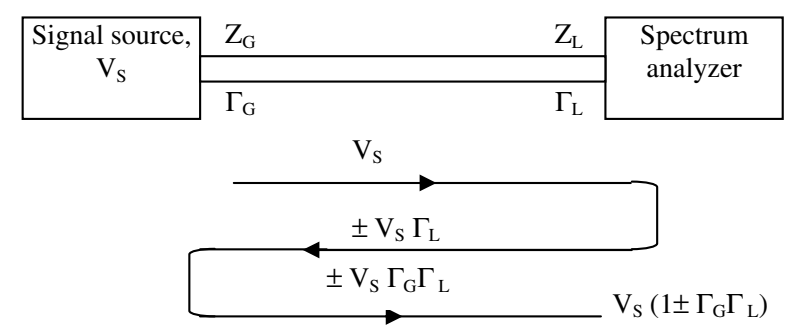

Fig. 1. Effect of mismatch in power measurements, $Z_{\mathrm{G}} \neq Z_{\mathrm{L}}$.

coefficient will give more precise data on the mismatch uncertainty while considering the overall uncertainty in the measurements.

Today, a vector network analyzer (VNA) system is easily available with the calibration labs and measurement of the complex reflection coefficient is possible. To use the complex reflection coefficient measurements, the exact electrical length of all cable and adaptors in the system should be known, and should remain constant over the time, temperature and many connect-disconnect cycles. These constraints are not possible and would require more VNA measurements to verify stability of phases. Also if one calculates the corrections for the mismatch loss or error, the uncertainties associated with the calculation parameters will contribute to the mismatch uncertainty. So, instead of performing the accurate measurements to have imprecise knowledge of this system used, adding a further term to uncertainty budget as mismatch uncertainty is recommended. Formulas for estimating mismatch uncertainty are derived in terms of reflection coefficients, VSWRs and $S$-parameters and evaluated earlier in [19-23]. These formulas were developed in accordance to the measurement parameter, its measuring system and the system for measuring the components of formulas. Selection of the mismatch uncertainty formula requires knowledge of the available formulas, the way of implementation and precise measurement of its components. The present paper addresses all these requirements and would help the technologists directly involve in RF calibration at any level.

\section{Expressions for the mismatch uncertainty when measuring RF power}

\subsection{Simple or direct power measurement}

If a signal source is connected to the spectrum analyzer or power meter for the power measurement as shown in Figure 1 and suppose the output impedance of the source $Z_{G}$ is not the same as of the analyzer $Z_{L}$. Due to the mismatch, a standing wave voltage will be generated as the signal reflects from the load (analyzer).

In the present case, the maximum measurement error due to mismatch can be found in terms of reflection coefficients. If the magnitudes of the reflection coefficient are used, the resulting formula for maximum mismatch error 
would be as per [5],

$$
M=1-\frac{1}{\left(1 \pm\left|\Gamma_{G}\right|\left|\Gamma_{L}\right|\right)^{2}}
$$

where $\left|\Gamma_{G}\right|$ is the reflection coefficient magnitude of source (generator), $\left|\Gamma_{L}\right|$ is the reflection coefficient magnitude of load (analyzer). These magnitudes can easily derive from the measured $V S W R$ in case.

The uncertainty limits in the mismatch error is given by in decibels $(\mathrm{dB})$,

$$
\text { Uncertainty limits }(\mathrm{dB})=20 \log _{10}\left|1 \pm \Gamma_{G} \Gamma_{L}\right|
$$

Max uncertainty limits $(\mathrm{dB})=20 \log _{10}\left|1+\Gamma_{\mathrm{G}} \Gamma_{\mathrm{L}}\right|$

Min uncertainty limits $(\mathrm{dB})=20 \log _{10}\left|1-\Gamma_{\mathrm{G}} \Gamma_{\mathrm{L}}\right|$.

For an example, if the source $V S W R$ is $<2.2: 1$ and the load $V S W R$ is $<1.8: 1$ at $1 \mathrm{GHz}$, the mismatch can be calculated as follows:

$$
\begin{aligned}
|\Gamma|_{G} & =\frac{\text { Source VSWR-1 }}{\text { Source VSWR+1 }}=\frac{2.2-1}{2.2+1}=0.375 \\
|\Gamma|_{L} & =\frac{\text { Load VSWR }-1}{\text { Load VSWR }+1}=\frac{1.8-1}{1.8+1}=0.286 .
\end{aligned}
$$

Max uncertainty limits in power $(\mathrm{dB})=20 \log _{10} \mid 1+$ $\Gamma_{\mathrm{G}} \Gamma_{\mathrm{L}} \mid=+0.884$.

Min uncertainty limits in power $(\mathrm{dB})=20 \log _{10} \mid 1-$ $\Gamma_{\mathrm{G}} \Gamma_{\mathrm{L}} \mid=-0.984$.

In a relative percentage,

Uncertainty limits in power $(\%)=$

$$
100 \times\left|\left(1 \pm\left|\Gamma_{G}\right|\left|\Gamma_{\mathrm{L}}\right|\right)^{2}-1\right| .
$$

Max uncertainty limits in power $(\%)=+22.58 \%$. Min uncertainty limits in power $(\%)=-20.28 \%$.

Uncertainty limits in voltage $(\%)=$

$$
100 \times\left|\Gamma_{\mathrm{G}}\right|\left|\Gamma_{\mathrm{L}}\right|= \pm 10.72 \% .
$$

In general power measurement system, for the calibration of power sensor or RF transfer standard connected to the compatible power meter, the calibration factor is defined as $[11,17,24]$,

$$
K=\frac{P_{D U T}}{P_{a p p}}\left(\frac{\left|1-\Gamma_{G} \Gamma_{L}\right|^{2}}{1-\left|\Gamma_{L}\right|^{2}}\right)
$$

where:

$P_{D U T}=$ power measured by the power sensor $(\mathrm{DUT})$ to be calibrated;

$P_{a p p}=$ applied power from the generator;

$\left|\Gamma_{G}\right|=$ the reflection coefficient magnitude of source (generator);

$\left|\Gamma_{L}\right|=$ the reflection coefficient magnitude of load (power sensor along with meter).
Here the factor $\left(1-\left|\Gamma_{L}\right|^{2}\right)$ is the calculable mismatch error and the limits of mismatch uncertainty are given by $\pm 2\left|\Gamma_{G}\right|\left|\Gamma_{L}\right|$. The mismatch uncertainty is type B uncertainty and having a $U$-shaped distribution due to a cosine function $[7,17]$. This means that the uncertainty of the mismatch is more likely to occur near the limits than in the center. So a divisor value $\sqrt{ } 2$ is being used to get the standard uncertainty (1-sigma factor) due to mismatch in the uncertainty analysis. The standard uncertainty of the mismatch is given by,

Standard mismatch uncertainty (linear) $=$

$$
\frac{2\left|\Gamma_{G}\right|\left|\Gamma_{L}\right|}{\sqrt{2}}=1.414\left|\Gamma_{G}\right|\left|\Gamma_{L}\right|
$$

Mismatch uncertainty for power in $\mathrm{dB}, M P(\mathrm{~dB})$ may be expressed as,

$$
M P(\mathrm{~dB})=8.686\left|\Gamma_{G}\right|\left|\Gamma_{L}\right| .
$$

Standard mismatch uncertainty $(\mathrm{dB})=$

$$
\frac{8.686\left|\Gamma_{G}\right|\left|\Gamma_{L}\right|}{\sqrt{2}}=6.143\left|\Gamma_{G}\right|\left|\Gamma_{L}\right| .
$$

Since the reflection coefficients are complex quantities composed of magnitude and phase components, the Gamma correction requires the full vector data to get the actual calibration factor $K^{\prime}$ in equation $(7)[7,17]$,

$$
\begin{aligned}
K^{\prime}= & \frac{P_{D U T}}{P_{a p p}} \\
& \times\left(\frac{\left(1-2\left|\Gamma_{G}\right|\left|\Gamma_{L}\right| \cos \left(\theta_{G}+\theta_{L}\right)+\left|\Gamma_{G}\right|^{2}\left|\Gamma_{L}\right|^{2}\right)}{1-\left|\Gamma_{L}\right|^{2}}\right)
\end{aligned}
$$

where:

$\theta_{L}=$ phase of reflection coefficient for the power sensor (DUT) to be calibrated;

$\theta_{\mathrm{G}}=$ phase of reflection coefficient for the generator.

Equations (7) and (9) are presenting the contribution of mismatch only and other factors effective efficiency, power drift, linearity, etc., are required to estimate combined standard uncertainty for calibration factor which is out of scope for the present paper. With the help of VNA, the complex $\Gamma$ can be measured in terms of magnitude and phase; accordingly equation (8) will modified for mismatch uncertainty for power in $\mathrm{dB}$ as,

$$
M P_{m p}=8.686\left|\Gamma_{G}\right|\left|\Gamma_{L}\right| \cos \left(\theta_{G}+\theta_{L}\right) .
$$

Using Source's VSWR only, equation (10a) will become

$$
M P_{m p}=8.686\left|\Gamma_{G}\right|\left|\Gamma_{L}\right| \cos \theta_{L}
$$

and in terms of real and imaginary components if $\Gamma_{L}=$ $p+j q$, the mismatch uncertainty can be written as,

$$
M P_{r i}=8.686\left(\left|\Gamma_{G}\right| \sqrt{p^{2}+q^{2}}\right) .
$$




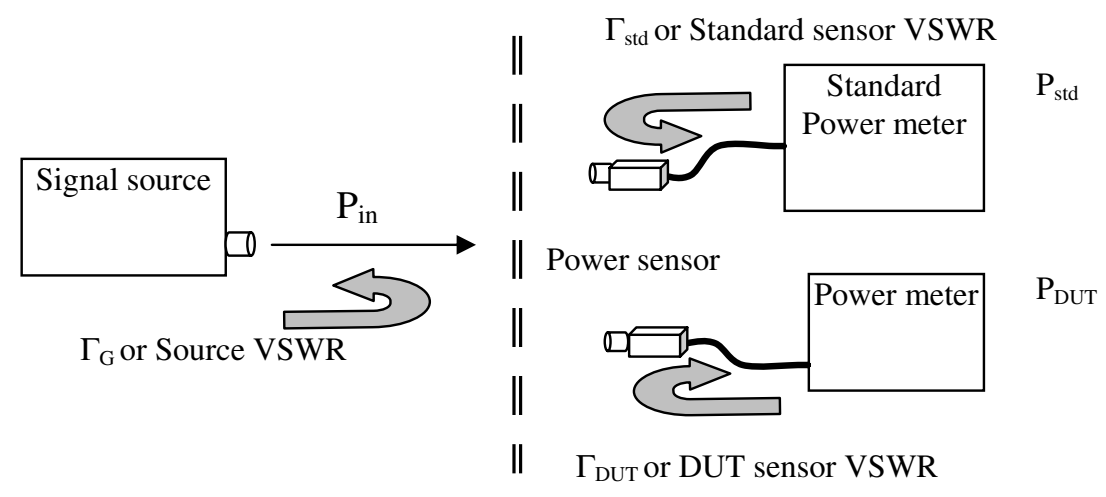

Fig. 2. Differential or null comparison power measurement.

\subsection{Differential or null comparison power measurement}

In an actual practice, the calibration factor of the sensor under calibration is evaluated in null comparison to the standard or reference power sensor and meter as shown in Figure 2. Thus the factors to contribute in total mismatch are the reflection coefficients of the signal generator, standard power sensor and the sensor under calibration.

Equation (7) can be modified to give the actual mismatch contribution as follows $[7,9,10]$,

$$
K^{\prime \prime}=\frac{P_{D U T}}{P_{s t d}}\left(\frac{\left|1-\Gamma_{G} \Gamma_{D U T}\right|^{2}}{\left|1-\Gamma_{G} \Gamma_{s t d}\right|^{2}}\right) \frac{1-\left|\Gamma_{s t d}\right|^{2}}{1-\left|\Gamma_{D U T}\right|^{2}}
$$

where:

$P_{\text {std }}=$ power applied measured on the reference power meter;

$\left|\Gamma_{s t d}\right|=$ the reflection coefficient magnitude of standard power sensor along with meter;

$\left|\Gamma_{D U T}\right|=$ the reflection coefficient magnitude of DUT power sensor along with meter.

Equation (10) can also be expanded similar to equation (8) to estimate the correct $K^{\prime \prime}$ as the vector measurements are easily realized using VNA systems for DUT and standard sensors. The replacements will be the complex reflection coefficients including the respective phase components. Now as the frequency ranges extend other than $50 \mathrm{MHz}$, the mismatch uncertainties are calculated at every frequency points and vector combined with reference measurements at $50 \mathrm{MHz}$ to evaluate $K$. Thus in the uncertainty budget, the total mismatch is contributed from the two factors of four reflection coefficients as each for standard sensor and DUT sensor at $50 \mathrm{MHz}\left(f_{1}\right)$ and the measurement frequency $\left(f_{2}\right)$ using the basic formula given by equation (13) as given in $[7,9,10]$. The expression for the mismatch uncertainty for null comparison power measurement $M N P$ is modified as,

$$
\begin{aligned}
M N P= & 8.686\left(\left\langle\left|\Gamma_{G}\right|\left(\left|\Gamma_{D U T}\right|+\left|\Gamma_{S t d}\right|\right)\right\rangle_{f_{1}}\right. \\
& \left.+\left\langle\left|\Gamma_{G}\right|\left(\left|\Gamma_{D U T}\right|+\left|\Gamma_{S t d}\right|\right)\right\rangle_{f_{2}}\right) .
\end{aligned}
$$

In terms of complex quantities, we can have the following formulas similar to equations (10) and (11),

$$
\begin{aligned}
& M N P_{m p}=8.686\left(\left\langle\left|\Gamma_{G}\right|\left(\left|\Gamma_{D U T}\right| \cos \theta_{D U T}+\left|\Gamma_{S t d}\right| \cos \theta_{S t d}\right)\right\rangle_{f_{1}}\right. \\
& \left.\quad+\left\langle\left|\Gamma_{G}\right|\left(\left|\Gamma_{D U T}\right| \cos \theta_{D U T}+\left|\Gamma_{S t d}\right| \cos \theta_{S t d}\right)\right\rangle_{f_{2}}\right)
\end{aligned}
$$

and in terms of real and imaginary components,

$$
\begin{gathered}
M N P_{r i}=8.686\left(\left\{\left|\Gamma_{G}\right|\left(\sqrt{p^{2}+q^{2}}+\sqrt{r^{2}+s^{2}}\right)\right\}_{f_{1}}\right. \\
\left.+\left\{\left|\Gamma_{G}\right|\left(\sqrt{p^{2}+q^{2}}+\sqrt{r^{2}+s^{2}}\right)\right\}_{f_{2}}\right)
\end{gathered}
$$

where $\Gamma_{D U T}=p+j q$ and $\Gamma_{S t d}=r+j s$.

\section{Expressions for the mismatch uncertainty when measuring microwave attenuation}

Any two-port component like an attenuator is characterized in terms of four coefficients, input and output reflection coefficients $\left(S_{11}, S_{22}\right)$, forward and reverse transmission coefficients $\left(S_{21}, S_{12}\right)$ in terms of $S$-parameters as shown in Figure 3.

In any attenuation measurement system based on either substitution or ratio technique, the attenuator under calibration is connected between the source and load ports, which results the insertion loss due to insertion of attenuator in the perfectly matched transmission line. This is because of the mismatch errors referring to the source and load sides of the measurement system. Normally instead of the attenuation, one measures the insertion loss using such system as given below [7, 17,21],

Insertion $\operatorname{loss}(\mathrm{dB})=20$

$$
\begin{array}{r}
\times \log _{10} \frac{\left|\left(1-\Gamma_{G} S_{11}\right)\left(1-\Gamma_{L} S_{22}\right)-\Gamma_{G} \Gamma_{L} S_{21} S_{12}\right|}{\left|S_{21}\right|\left|1-\Gamma_{G} \Gamma_{L}\right|} \\
=A(\mathrm{~dB})+M i \operatorname{serr}(\mathrm{dB})
\end{array}
$$

where attenuation to be measured is given by,

$$
A(\mathrm{~dB})=20 \log _{10} \frac{1}{\left|S_{21}\right|} .
$$




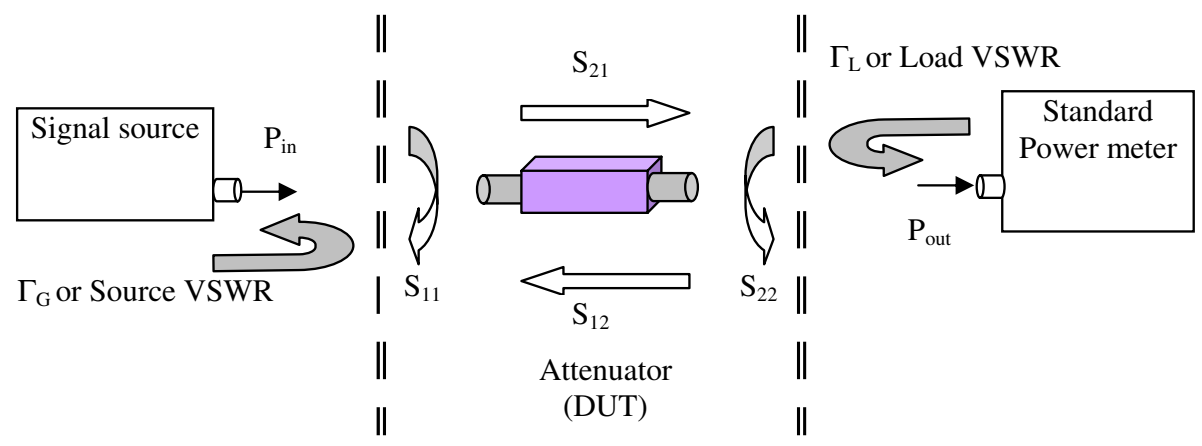

Fig. 3. (Color online) Simple attenuation measurement.

$$
\begin{gathered}
\text { Mismatch error }(\mathrm{dB})=10 \log _{10} \frac{\left|1-\left(\Gamma_{G} S_{11}+\Gamma_{L} S_{22}+\Gamma_{G} \Gamma_{L} S_{21} S_{12}\right)+\Gamma_{G} \Gamma_{L} S_{11} S_{22}\right|^{2}}{\left|1-\Gamma_{G} \Gamma_{L}\right|^{2}} \\
M A_{T}=\frac{1 \pm\left(\left|\Gamma_{G} S_{11}\right|+\left|\Gamma_{L} S_{22}\right|+\left|\Gamma_{G} \Gamma_{L} S_{11} S_{22}\right|+\left|\Gamma_{G} \Gamma_{L} S_{21} S_{12}\right|\right)}{1 \mp\left|\Gamma_{G}\right|\left|\Gamma_{L}\right|} \\
M A_{T}(\mathrm{~dB})=20 \log _{10} \frac{1 \pm\left(\left|\Gamma_{G} S_{11}\right|+\left|\Gamma_{L} S_{22}\right|+\left|\Gamma_{G} \Gamma_{L} S_{11} S_{22}\right|+\left|\Gamma_{G} \Gamma_{L} S_{21} S_{12}\right|\right)}{1 \mp\left|\Gamma_{G}\right|\left|\Gamma_{L}\right|}
\end{gathered}
$$

Thus in general, the mismatch contributors in the attenuation measurements are the source reflection coefficient (or $V S W R$ ), load reflection coefficient (or $V S W R$ ) and four $S$ parameters of the attenuator under calibration. The few expressions given in the next section are further modified for the calculation from measured $S$-parameters in various forms like complex and linear compared to the earlier ones in case of the fixed and variable attenuators.

\subsection{For flat attenuation, i.e., fixed attenuator}

The difference between the measured insertion loss and attenuation is termed as the mismatch error and is defined in terms of complex independent variables as $[6,8,19]$,

$$
\text { see equation (18) above }
$$

or,

$$
\begin{aligned}
& \text { Mismatch error }(\mathrm{dB})=20 \\
& \qquad \times \log _{10} \frac{\left|\left(1-\Gamma_{G} S_{11}\right)\left(1-\Gamma_{L} S_{22}\right)-\Gamma_{G} \Gamma_{L} S_{21} S_{12}\right|}{\left|1-\Gamma_{G} \Gamma_{L}\right|}
\end{aligned}
$$

where $\Gamma_{\mathrm{L}}$ and $\Gamma_{\mathrm{G}}$ will be act as effective load reflection coefficient and effective source reflection coefficient for $S_{21}$ measurement at the insertion planes and vice-versa for $S_{12}$ respectively. $S_{11}, S_{12}, S_{21}$ and $S_{22}$ are the four $S$ parameters of attenuator under calibration.

Total uncertainty contribution for attenuation $M A_{T}$ due to mismatch error for two-port component i.e. for fixed attenuator can be derived from equation (19), when only magnitude is known (linear), applicable in case of low attenuation values $(<10 \mathrm{~dB})[17,19,21]$.

See equation (20) above.
The distribution for $M A_{T}(\mathrm{~dB})$ would be a normal distribution as it has been proved that combination of four independent $U$-shaped distributions is nearly a normal distribution [24]. If $S_{11}, S_{22}, \Gamma_{\mathrm{G}}$ and $\Gamma_{\mathrm{L}}$ are extremely small, i.e. $\ll 1$, in $\mathrm{dB}$ for almost the matched conditions, the maximum mismatch uncertainty for attenuation would be evaluated by [20],

$$
\begin{aligned}
M A(\mathrm{~dB})= & 8.686\left[\left|\Gamma_{G}\right|\left|S_{11}\right|+\left|\Gamma_{L}\right|\left|S_{22}\right|\right. \\
& \left.+\left|\Gamma_{G} \Gamma_{L}\right|\left(1+\left|S_{21} S_{12}\right|\right)\right] .
\end{aligned}
$$

If the reflection coefficient measurements are performed in terms of magnitude and phase by VNA systems and the phase of $\Gamma_{\mathrm{G}}$ is unknown, the mismatch uncertainty can be evaluated in $\mathrm{dB}$ (applicable for high attenuation values, $>10 \mathrm{~dB})$ given in $[20,21,24]$.

$$
\begin{gathered}
M A_{m p}(\mathrm{~dB})=8.686\left(| \Gamma _ { G } | | \Gamma _ { L } | \left\langle\cos \left(\phi \Gamma_{L}\right)-\left|S_{21}\right|\left|S_{12}\right|\right.\right. \\
\left.\times \cos \left(\phi \Gamma_{L}+\phi_{12}+\phi_{21}\right)\right\rangle-\left|\Gamma_{G}\right|\left|S_{11}\right| \cos \left(\phi_{11}\right) \\
\left.-\left|\Gamma_{L}\right|\left|S_{22}\right| \cos \left(\phi \Gamma_{L}+\phi_{22}\right)\right) .
\end{gathered}
$$

When the measured $S$-parameters are in terms of real and imaginary components and defined as $S_{11}=a+j b, S_{22}=$ $e+j f, S_{21}=k+j l, S_{12}=p+j q$, the mismatch uncertainty will be,

$$
\begin{array}{r}
M A_{r i}(\mathrm{~dB})=8.686\left(\left|\Gamma_{G}\right| \sqrt{a^{2}+b^{2}}+\left|\Gamma_{L}\right| \sqrt{e^{2}+f^{2}}\right. \\
\left.+\left|\Gamma_{G}\right|\left|\Gamma_{L}\right|\left(\sqrt{(p k-q l)^{2}+(p l-q k)^{2}}\right)\right) .
\end{array}
$$

If the calibration lab is having only VSWR measurement facility and attenuation is measured by Intermediate 


$$
M S A(\mathrm{~dB})=20 \log _{10} \frac{1 \pm\left(\left|\Gamma_{G} S_{11}\right|+\left|\Gamma_{L} S_{22}\right|+\left|\Gamma_{G} \Gamma_{L} S_{11} S_{22}\right|+\left|\Gamma_{G} \Gamma_{L} S_{21} S_{12}\right|\right)}{1 \pm\left(\left|\Gamma_{G} S_{11}^{\prime}\right|+\left|\Gamma_{L} S_{22}^{\prime}\right|+\left|\Gamma_{G} \Gamma_{L} S_{11}^{\prime} S_{22}^{\prime}\right|+\left|\Gamma_{G} \Gamma_{L} S_{21}^{\prime} S_{12}^{\prime}\right|\right)}
$$

frequency (IF) substitution technique, the mismatch uncertainty in terms of $V S W R$ values is expressed by [22-24],

$$
\begin{aligned}
M A_{\text {vswr }}(\mathrm{dB})= & \pm 2\left(\left(V S W R_{G}-1\right)\left(V S W R_{D U C I}-1\right)\right. \\
& \left.+\left(V S W R_{L}-1\right)\left(V S W R_{D U C O}-1\right)\right)
\end{aligned}
$$

where:

$V S W R_{G}: \quad$ measured $V S W R$ at the generator port;

$V S W R_{L}$ : measured VSWR at the load or detector port;

$V S W R_{D U C I}$ : measured $V S W R$ at the input port of the attenuator (DUC);

$V S W R_{D U C O}$ : measured VSWR at the output port of the attenuator (DUC).

\subsection{For incremental attenuation, i.e., step attenuator}

In the case of the calibration of step attenuator or variable attenuator, two setting values would be considered at each measurement frequency, one the initial value i.e. ' 0 ' $\mathrm{dB}$ and the final value, the attenuation level to be measured. So each attenuation value can be considered as one twoport attenuator is replaced by second two-port attenuator, and mismatch error for incremental attenuation is given by $[6,17,21]$,

Mismatch error $(\mathrm{dB})=20$

$$
\times \log _{10} \frac{\left|\left(1-\Gamma_{G} S_{11}\right)\left(1-\Gamma_{L} S_{22}\right)-\Gamma_{G} \Gamma_{L} S_{21} S_{12}\right|}{\left|\left(1-\Gamma_{G} S_{11}^{\prime}\right)\left(1-\Gamma_{L} S_{22}^{\prime}\right)-\Gamma_{G} \Gamma_{L} S_{21}^{\prime} S_{12}^{\prime}\right|}
$$

where:

$\Gamma_{\mathrm{G}}$ : effective source port match;

$\Gamma_{\mathrm{L}}$ : effective load match;

$S_{11}, S_{12}, S_{21}, S_{22}$ : scattering coefficients of the attenuator (at the attenuation level);

$S_{11}^{\prime}, S_{12}^{\prime}, S_{21}^{\prime}$, and $S_{22}^{\prime}$ : scattering coefficients of the two-port device at the initial state ' 0 ' $\mathrm{dB}$.

In this case, the incremental attenuation is defined by,

$$
A_{\text {incremental }}(\mathrm{dB})=20 \log _{10} \frac{1}{\left|S_{21}\right|}-20 \log _{10} \frac{1}{\left|S_{21}^{\prime}\right|} .
$$

Uncertainty due to mismatch for the step attenuator in $\mathrm{dB}, M S A(\mathrm{~dB})$ is defined as,

\section{See equation (27) above.}

If $S_{11}, S_{22}, \Gamma_{\mathrm{G}}$ and $\Gamma_{\mathrm{L}}$ are extremely small i.e. $\ll 0.5$ $(\approx 0.01)$ for well-matched system and DUT, i.e. attenuation measurement by VNA system, the maximum mismatch uncertainty in magnitude terms will be expressed as $[21,24]$,

$$
\begin{aligned}
& M S A(\mathrm{~dB})=8.686\left(\left|\Gamma_{G}\right|^{2}\left(\left|S_{11}^{\prime}\right|^{2}-\left|S_{11}\right|^{2}\right)+\left|\Gamma_{L}\right|^{2}\left(\left|S_{22}^{\prime}\right|^{2}\right.\right. \\
& \left.\left.-\left|S_{22}\right|^{2}\right)+\left|\Gamma_{G}\right|^{2}\left|\Gamma_{L}\right|^{2}\left(\left|S_{21}^{\prime}\right|^{2}\left|S_{12}^{\prime}\right|^{2}-\left|S_{21}\right|^{2}\left|S_{12}\right|^{2}\right)\right)^{1 / 2} .
\end{aligned}
$$

For high attenuation values, if the $S$-parameter values are in magnitude and phase components with no measurement of the phase of $\Gamma_{\mathrm{G}}$, the mismatch uncertainty in $\mathrm{dB}$ is given by [24],

$$
\begin{array}{r}
M S A_{m p}(\mathrm{~dB})=8.686\left(\left|\Gamma_{G}\right|\left\langle\left|S_{11}^{\prime}\right| \cos \left(\phi_{11}^{\prime}\right)-\left|S_{11}\right| \cos \left(\phi_{11}\right)\right\rangle\right. \\
+\left|\Gamma_{G}\right|\left|\Gamma_{L}\right|\left\langle\left|S_{21}^{\prime}\right|\left|S_{12}^{\prime}\right| \cos \left(\phi_{\Gamma_{L}}+\phi_{12}^{\prime}+\phi_{21}^{\prime}\right)-\left|S_{21}\right|\left|S_{12}\right|\right. \\
\left.\times \cos \left(\phi_{\Gamma_{L}}+\phi_{12}+\phi_{21}\right)\right\rangle+\left|\Gamma_{L}\right|\left\langle\left|S_{22}^{\prime}\right| \cos \left(\phi_{\Gamma_{L}}+\phi_{22}^{\prime}\right)\right. \\
\left.\left.-\left|S_{22}\right| \cos \left(\phi_{\Gamma_{L}}+\phi_{22}\right)\right\rangle\right) . \quad(29)
\end{array}
$$

Now consider, the representations for the measured complex $S$-parameters as,

$$
\begin{aligned}
& S_{11}=a+j b, S_{11}^{\prime}=c+j d, S_{22}=e+j f, S_{22}^{\prime}=g+j h, \\
& S_{21}=k+j l, S_{21}^{\prime}=m+j n, S_{12}=p+j q, S_{12}^{\prime}=r+j s
\end{aligned}
$$

$\Gamma_{\mathrm{G}}$ and $\Gamma_{\mathrm{L}}$ will be act as an effective load match and effective test port match respectively for $S_{12}$.

So, the mismatch uncertainty in terms of real and imaginary components can be evaluated by the following formula [21],

$$
\begin{aligned}
& M S A_{r i}(\mathrm{~dB})=8.686\left(\left|\Gamma_{G}\right| \sqrt{(a-c)^{2}+(b-d)^{2}}\right. \\
& +\left|\Gamma_{L}\right| \sqrt{(e-g)^{2}+(f-h)^{2}}+\left|\Gamma_{G}\right|\left|\Gamma_{L}\right| \\
& \left.\times \sqrt{(p k-q l-m r+n s)^{2}+(p l+q k-m s-n r)^{2}}\right) .
\end{aligned}
$$

Mismatch uncertainty in terms of the reflection coefficients for variable attenuator in $\mathrm{dB}$ is given by the following expression [22-24],

$$
\begin{aligned}
M S A_{\Gamma}(\mathrm{dB})= & 20 \log _{10}\left(1-\Gamma_{G}\left(\Gamma_{i 1}-\Gamma_{i 2}\right)-\Gamma_{L}\left(\Gamma_{o 1}-\Gamma_{o 2}\right)\right. \\
& \left.+\Gamma_{G} \Gamma_{L}\left(10^{-A_{1} / 10}-10^{-A_{2} / 10}\right)\right)
\end{aligned}
$$

where:

$\Gamma_{L}$ : load reflection coefficient at the detector port;

$\Gamma_{G}$ : generator (Source) reflection coefficient at the generator port;

$\Gamma_{i 1}$ and $\Gamma_{i 2}$ are the measured reflection coefficients of the variable attenuator for two settings of attenuator at the input port; 


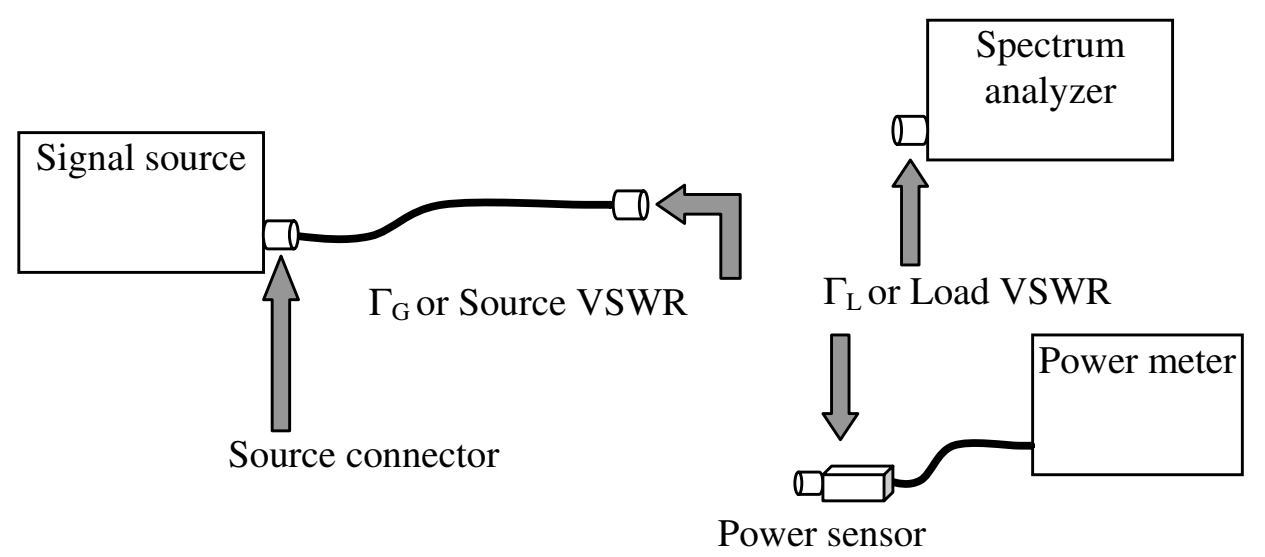

Fig. 4. Illustrations for $\Gamma$ or $V S W R$ measurement planes.

$\Gamma_{o 1}$ and $\Gamma_{o 2}$ are the measured reflection coefficients of the variable attenuator for two settings of attenuator at the output port;

$A_{1}$ and $A_{2}$ are the measured attenuation values of the variable attenuator for two settings of attenuator.

The reflection coefficient values $\Gamma_{G}, \Gamma_{L}, \Gamma_{D U T}, \Gamma_{S t d}$, etc. and the scattering coefficients used are subject to uncertainty in the above expressions, because they are obtained by the measurements. Their associated uncertainty should be certainly considered while calculating the mismatch uncertainty and it is suggested adding the uncertainty of the reflection coefficient in quadrature with its measured value [7]. For example, if $\Gamma_{\mathrm{L}}$ is $0.025 \pm 0.010$, the value of $\Gamma_{\mathrm{L}} \approx \sqrt{ }\left(0.025^{2}+0.010^{2}\right)=0.027$ should be used in the estimation of mismatch uncertainty.

\section{Best practices for limiting the effect of the mismatch}

This section discusses the measurement planes for complex reflection coefficient or $V S W R$ in measurement systems, techniques to reduce and control the mismatch uncertainty in brief. While completely eliminating mismatch loss in the components and system is nearly impossible, selecting quality components for use in a well-designed system can minimize the mismatch loss contributions by each component.

\subsection{Complex reflection coefficient or VSWR measurement plane}

In general, the knowledge of the output impedance of the RF source (source match) is important to allow users to estimate mismatch uncertainty contributions in their applications. However, the measurement techniques generally used for passive components cannot be used for an active leveled source. If those methods are used, they are likely to give erroneous misleading results because in operation, any reflections caused by a mismatch between the source and the load will be at the signal frequency, and the leveling circuit will detect the combined effect of the output signal and the reflection. Thus the methods appropriate for passive devices do not give appropriate conditions to correctly measure the equivalent source match. The measurement plane is very important to decide the place of measurement for source $V S W R$ measurement as the source is generally connected to the load (meter or analyzer) through a coaxial cable. So the $V S W R$ measurement should be performed at the other end of cable where the DUT has to attach. Better illustrations are given in Figure 4 for understanding the measurement planes.

The manufacturer provides the $V S W R$ of signal source measured in its off condition at the source connector as shown in Figure 2. As the actual value of $V S W R$ is not known for the source, the specified values of $V S W R$ are being used in the estimation under the assumption that they meet their specifications. For an accurate estimation for mismatch error and precision measurements, the actual value of $\Gamma$ or $V S W R$ is necessary due to its dependency over the operating frequency and applied power ranges. So, the user should measure $\Gamma_{G}$ or $V S W R_{G}$ at the cable end connected to the source (generator) to apply equations $(1)-(15)$ precisely. In practice, the reflection coefficient or $V S W R$ of the source is not measured in active mode due to power coming outside and its $V S W R$ is specified as a maximum value for the operating frequency range. The VSWR of the source can be measured and controlled with a suitable and calibrated attenuator.

\subsection{Reduction of the mismatch uncertainty}

It can be reduced to a great extent by using the following techniques $[7,25-28]$ :

\subsubsection{Inserting the attenuator pads}

If the signal to be measured is relatively large, placing a precision attenuator in the measurement path can minimize mismatch uncertainty. Reflected power due to the 
mismatch will be reduced on the returning to the source by the attenuator. If the matching attenuator characteristics are well known and very stable, inserting the attenuation will reduce the measurement uncertainty effectively. One disadvantage is that it also reduces the measurement range of attenuation and power. The mismatch uncertainty is $50 \%$ reduced using the precision fixed attenuator pads of values $10 \mathrm{~dB}$ at the source and detector ports, respectively in our substitution techniques based on the coaxial configuration.

\subsubsection{Use of stub tuners and matching network}

To minimize the mismatch uncertainty, stub tuners are used with isolators $(>15 \mathrm{~dB})$ at frequency higher than $1 \mathrm{GHz}$ and the matching networks are used at lower frequencies like with plasma deposition components. These systems are basically a combination of variable capacitors and inductors, which are tuned to match the components connected at the ends for $V S W R \leqslant 1.02$. However, these techniques are not required for the measurements by VNA systems. The multi-stub tuners are being used in waveguide substitution techniques and the mismatch uncertainty is reduced by a factor of 3 .

\subsubsection{Use of torque wrench and good quality cables}

Torque wrench applied an appropriate pressure on the connectors of components or cables in dealing with them. Thus their application in connecting the devices will reduce greatly the physical damage, so the mismatch uncertainty. We use a torque of $136 \mathrm{~N}-\mathrm{cm}$ on APC-7 and Type-N connectors. The low-loss cables $(1.5 \mathrm{~dB} / \mathrm{m})$ with low $V S W R(\leqslant 1.05)$ connectors are also benefitted in terms of low attenuation and accurate measurement.

\subsection{Control on the mismatch uncertainty}

Practicing few precautionary steps also help in controlling the mismatch uncertainty up to a certain level [7,25-28].

\subsubsection{Regular visual inspection and cleaning of connectors}

The operator should make inspection and clean the connectors by Isopropanol with cotton swabs in the regular interval and before using. This will keep any dust landing or metal degrading away. We observe every connector whether APC-7 or Type-N for any kind of defect before using in practice.

\subsubsection{Use of connector gauge kits}

The connector gauge kits measures the pin depths of the connectors. The use of such kits helps in finding of any capacitance or additional pressure on the connector pin or slot while connecting them. The operator can replace the connector if finds so. We also send the connector gauge kits for calibration in intervals, which helps us to precisely find the pin depth as per IEEE standard 287.

\subsubsection{Self-experience}

The implementation and follow up of the above steps by a well experience metrologist or technical operator greatly eliminates any additional source of mismatch error and increase the life of the system and components. The connector repeatability also indicates a measure of the handling and maintenance of the connectors by the operator.

\section{Conclusion}

This paper describes the mismatch uncertainty as an important uncertainty contributor for RF calibration with its advanced methodology being used at the calibration labs. The fundamental cause and evaluation of this uncertainty component has been given in detail for many cases. The formulas of estimating mismatch uncertainty are summarized for their appropriate and suitable applications in the attenuation and power calibrations. The mismatch uncertainty formulas are presented and modified applicable for the precision measuring system existing in the calibration labs. The methods are also discussed for the reduction and control of the mismatch uncertainty to an insignificant level. Every time mismatch component is measured against the ideal value $50 \Omega$ in terms of $\Gamma, V S W R, R L$ and scattering parameters $\left(S_{n n}\right)$, so to estimate this uncertainty precisely, the calibration labs should establish and maintain the standards and calibration facilities for reflection coefficient or VSWR parameters. In other words, the accredited calibration range of these parameters decides the traceable range of power and attenuation parameters while estimating the mismatch uncertainty from the measured values. Thus the implementation of precise mismatch uncertainty estimation requires traceability of one-port measurements in accordance to ISO 17025:2005 and GUM document.

Acknowledgements. This paper describes the work of many great people and could not have been written without their enormous contributions in this field earlier. Thus the authors would like to pay tribute to the previous researchers in this field. The work has been supported by Director, NPLI and Head, Electrical Standards, NPLI under the project NWP-45.

\section{References}

1. E. Hund, Microwave Communication - Components and Circuits (McGraw-Hill Book Company, 1987)

2. G. Kennedy, Electronic Communication Systems (McGraw-Hill Education, Ltd., India, 1994) 
3. R.W. Beatty, Mismatch errors in the measurement of ultrahigh frequency and microwave variable attenuators, Journal of Research National Bureau of Standards 52, 7-9 (1954)

4. G.E. Schafer, A.Y. Rumfelt, Mismatch errors in the cascade-connected variable attenuators, IRE Transactions of Microwave Theory and Techniques 7, 447-453 (1959)

5. Microwave mismatch error analysis, Hewlett-Packard Application note 56, 1967

6. R.W. Beatty, Microwave Attenuation measurements and standards, NBS Monograph 97 (1967)

7. Microwave measurements, edited by R. Collier, D. Skinner (IET Publication, 2009)

8. D.L. Hollway, F.P. Kelly, A standard attenuator and the Precise measurement of Attenuation, IEEE Trans. Instrum. Meas. 13, 33-44 (1964)

9. S. Whitacre, Dee Humpherys, Low level power measurement techniques and accuracy, HP RF \& Microwave measurement symposium and exhibition (USA, 1968)

10. G.F. Engen, Recent development in the field of microwave power measurements at the National Bureau of Standards, IRE Trans. Instrum. 3, 304-306 (1958)

11. R.W. Beatty, Coaxial Power, impedance and attenuation calibration methods at NBS, Microwave J., 65-75 (1968)

12. ISO, ISO/IEC 17025, General requirements for the competence of testing and calibration laboratories (2005)

13. Guidelines on the evaluation and expression of the measurement uncertainty, Singapore Institute of Standards and Industrial Research (1995)

14. Guide to the expression of uncertainty in measurement, Geneva, International Organization for Standardization (1993)

15. EA Guidelines on the Evaluation of Vector Network Analyzers (VNA), Publication reference EUROMET/cg12/v0.1 (2007)
16. G.H. Bryant, Principles of microwave measurements (Peter Peregrinus Ltd., 1988)

17. F.L. Warner, Microwave Attenuation measurement (Peter Peregrinus Ltd., 1977)

18. A.E. Bailey, Microwave measurements (IEE Publication, 1989)

19. Horst Bayer, F.L. Warner, R.W. Yell, Attenuation and Ratio - National Standards, Proc. IEEE 74, 46-59 (1986)

20. H. Bayer, An error analysis for the RF attenuation measuring equipment of the PTB applying the power method, Metrologia 11, 43-51 (1975)

21. F.L. Warner, New expression for mismatch uncertainty when measuring microwave attenuation, IEE Proc. 127, 66-69 (1980)

22. R.S. Yadava, R. Swarup, K. Chandra, Technical report on Audio frequency substitution technique for measurement of Microwave attenuation, Report No. MWA-1, 1983

23. R. Swarup, R.S. Yadava, K. Chandra, Technical report on Microwave attenuation measurement by IF substitution technique, Report No. MWA-2, 1983

24. I.A. Harris, F.L. Warner, Re-examination of mismatch uncertainty when measuring microwave power and attenuation, IEE Proc. 128, 35-41 (1981)

25. B.O. Weinschel, Standardization of precision coaxial connectors, Proc. IEEE 55, 923-931 (1967)

26. Coaxial connectors in Radio frequency and microwave measurements, NAMAS, NIS 4303 (1991), Vol. 1

27. D. Skinner, ANAMET Connector guide-Guidance on using Coaxial connectors in measurement, ANAMET Report 032, 2001

28. IEEE Std. $287^{\mathrm{TM}}-2007$, IEEE Standard for Precision coaxial connectors (DC to $110 \mathrm{GHz}$ ) (IEEE Instrumentation and Measurement Society, USA, 2007) 